\title{
Chitosan microgel: Effect of cross-linking density on pH-dependent release
}

\author{
Mi Kyoung Kang*, Sung Kyeong Hong*, Yong Chang Seo**, Young Ock Kim***, \\ Hyeon Yong Lee*, and Jin-Chul Kim*,"

\begin{abstract}
*Division of Biotechnology \& Bioengineering and Institute of Bioscience and Biotechnology, Kangwon National University, 192-1, Hyoja-2dong, Chunchon, Kangwon 200-701, Korea **Medical \& Bio-Material Research Center and Department of Biomaterials Engineering, Kangwon National University, Chuncheon 200-701, Korea ****Department of Herbal Science, RDA, Eumseong 369-873, Korea
\end{abstract} (Received 3 March 2011 • accepted 18 May 2011)

\begin{abstract}
Chitosan microgels were prepared by a spray-drying method using glutaraldehyde (GA) as a cross-linker. Two kinds of microgels, so-called soft microgel and hard microgel, were prepared using a chitosan to GA ratio of $1: 0.08$, and $1: 0.67$, respectively. The surfaces of hard microgels were more even that those of soft microgels. The swelling ratio, a measure of degree of swelling, of the soft microgel was $\mathrm{pH}$-sensitive, and it decreased from 1,765\% to $1,230 \%$, when the $\mathrm{pH}$ increased from 4.0 to 9.0. The deprotonation of amino groups of chitosan could account for the decrease in swelling ratio. The swelling ratio of hard microgels was almost invariable in response to $\mathrm{pH}$ change (4.0 to 9.0), and it was much less than that of soft microgels, possibly due to the high cross-linking density. The degree of release from the soft microgels decreased from $65 \%$ to $12 \%$ when the $\mathrm{pH}$ of medium increased from 4.0 to 6.0. FITC-dextran would readily diffuse out of the microgel, possibly due to the larger meshes of the microgels in a strong acidic condition (e.g., $\mathrm{pH} 4.0$ ). However, the degree of release increased from $12 \%$ to $82 \%$ when the $\mathrm{pH}$ of medium increased from 6.0 to 9.0 . In this $\mathrm{pH}$ range, the fluorescence dye is believed to be released mainly by a squeezing-out.
\end{abstract}

Key words: Chitosan Microgels, Spray Drying, pH-sensitive Swelling Ratio, pH-sensitive Release

\section{INTRODUCTION}

To maximize bioavailability, a drug is required to be released from a carrier at a specific site or under a specific condition. Drug carriers which release their contents in response to external stimuli, such as changes in $\mathrm{pH}$ [1-4], temperature [5-7], light [8-10] and electromagnetic radiation [11,12], have been developed. Due to the versatile applications, much attention has been paid to $\mathrm{pH}$-sensitive carriers. $\mathrm{pH}$-sensitive vesicles were proposed as a carrier for cytoplasmic delivery of a drug via endocytosis [13-15], and they were also known to be applicable to the treatment of cancer, since endosome and cancer tissue are in weak acidic conditions. Titrable lipids such as cholesteryl hemisuccinate (CHEMS) and oleic acid were included in the phosphorlipid bilayers to achieve an extensive release under an acidic condition. Another method to achieve a $\mathrm{pH}$-sensitive release is to modify the surface of vesicles with titrable polymers such as chitosan [16,17], protenoid [18], and poly( $N$-isopropylacrylamide-comethacrylic acid-co-octadecylacrylate) [19]. On the other hand, alginate bead coated with chitosan exhibited a $\mathrm{pH}$-sensitive release and it might be used as a carrier for the oral delivery of protein drugs [16]. Under gastric condition, chitosan will be protonated and it will strongly interact with the surface of the alginate bead. As a result, a condensed skin layer of chitosan will be formed, suppressing a release from the beads. Under intestinal condition, chitosan layer will swell due to a slackened interaction with alginate, resulting in an enhanced release. Recently, microgels have attracted great attention as a par-

To whom correspondence should be addressed.

E-mail: jinkim@kangwon.ac.kr ticulate drug delivery carrier. The drugs can be embedded in microgel interior network and they can be released out due to the open network of the microgel structure. Their average diameters are in the range between $50 \mathrm{~nm}$ and $5 \mathrm{~mm}$ [20]. They have a large amount of water, adjustable chemical and mechanical properties, and biocompatibility. Microgel is prepared by various methods such as waterin-oil heterogeneous gelation, continuous extrusion, microfluidic preparation, precipitation in water, supramolecular self-complexation and spray drying [21]. Chitosan-based microgels are designed by crosslinking with chitosan derivative, $\mathrm{N}-[$ (2-hydroxy-3-trimethylammonium)propyl]chitosan chloride and sodium tripolyphosphate. At low $\mathrm{pH}$ (e.g., $\mathrm{pH}$ 5.0), chitosan microgels swell due to repulsion between the protonated amino groups and exhibit a faster release of the drug. As a result, the microgel is efficacious in killing Hela cells and was proposed to be used as a carrier for intracellular targeted delivery [22]. As a biomaterial, chitosan is known to be non-toxic and biocompatible $[23,24]$. It is mainly composed of glucosamine and obtained from deacetylating chitin, which is a polysaccharide abundant in the exoskeleton of shellfish such as shrimp, lobster, and crabs. It has been used as a pH-sensitive excipient for a $\mathrm{pH}$-sensitive carrier. In this study, the chitosan microgels were prepared by a spray-drying method using glutaraldehyde as a cross-linker. The effect of the amount of cross-linker on the surface topology, the $\mathrm{pH}$-dependent swelling, and the $\mathrm{pH}$-dependent release was extensively studied.

\section{EXPERIMENTAL}

\section{Materials}

Fluorescein isothiocyanate-dextran (FITC-dextran, MW. 40,000), 
chitosan (low molecular weight, degree of deacetylation: 91.8\%), and Tris(hydroxymethyl)-aminomethane were provided by SigmaAldrich, inc. (Saint Louis, MO, USA). Glutaraldehyde (GA) was provided by Merck KGaA (Darmstadt, Germany). 2-( $N$-morpholino)ethane sulfonic acid (MES) was purchased from Biopure (Burlington, Ontario, Canada). Acetic acid was obtained from Junsei Chemical Co., Ltd (Tokyo, Japan). $N$-(2-hydroxyethyl)piperazine- $N$ '-(2ethanesulfonic acid) (HEPES) was provided by USB Corporation.

\section{Preparation of Chitosan Microgels}

Chitosan solution was prepared by dissolving $10 \mathrm{~g}$ of chitosan in $1 \mathrm{~L}$ of acetic acid solution (1\%). GA, a cross-linking agent, was added to the chitosan solution so that the ratio of chitosan to GA is $1: 0,1: 0.08$, and $1: 0.67$. After stirring the mixture solutions for $1 \mathrm{~min}$, microgels were prepared by spray-drying the solutions using a spray dryer (BUCHI B-290, Switzerland). The inlet temperature was $160{ }^{\circ} \mathrm{C}$, aspiration was $80 \%$, pumping was $15 \%$ (corresponding to the feed flow rate of $4 \mathrm{ml} / \mathrm{min}$ ), and the air flow rate was 6.9 $\mathrm{L} / \mathrm{min}$. Unreacted chitosan and GA were leached out of microgels by washing them for $5 \mathrm{hr}$ in distilled water (adjusted to $\mathrm{pH} \mathrm{4.0)}$ ). The washed microgels were retrieved by centrifuging the suspension and they were lyophilized in a freezer-drier (TFD 5508). The microgels prepared at chitosan to GA ratio of $1: 0.08$, and $1: 0.67$ are to be termed as "soft microgels" and "hard microgels," respectively.

\section{Fourier Transform Infrared Spectroscopy (FTIR) of Micro-} spheres and Microgels

FTIR spectra of uncrosslinked microspheres, soft microgels and hard microgels in $\mathrm{KBr}$ pellets were taken on a Perkin Elmer Fourier transform infrared spectrophotometer instrument (EXCALIBER Series, U.S.A.).

\section{Scanning Electron Microscopy (SEM) of Microspheres and Microgels}

Freeze-dried soft and hard microgels were put on metal stubs, and they were coated with gold and observed in a scanning electron microscope (Jeol JSM-840A). In parallel, uncrosslinked microspheres were investigated along with the microgels.

\section{Swelling Ratio of Microgels}

The swelling ratios of soft microgels and hard microgels were measured at room temperature with varying $\mathrm{pHs}$. The microgels of around $0.2 \mathrm{~g}$ were put into $10 \mathrm{ml}$ of buffer solution contained in a $50 \mathrm{ml}$ beaker. MES was used for $\mathrm{pH}$ 4.0, $\mathrm{pH}$ 5.0, HEPES for $\mathrm{pH}$ 6.0, $\mathrm{pH}$ 7.0, $\mathrm{pH}$ 8.0, and Tris buffer for $\mathrm{pH}$ 9.0. The suspensions were gently stirred for $4 \mathrm{hr}$ and the microgels were retrieved by a centrifugation method. The swollen microgels were weighed and the swelling ratios were determined as follows [25].

Swelling ratio $(\%)=[($ wet weight - dry weight $) /$ dry weight $] \times 100$

\section{Loading of FITC-dextran in Microgels}

FITC-dextran, a fluorescence marker, was loaded in microgels. FITC-dextran, $35 \mathrm{mg}$ was dissolved in $60 \mathrm{ml}$ of HEPES buffer (3 $\mathrm{mM}, \mathrm{pH}$ 6). Dry microgels, $2 \mathrm{~g}$, were soaked in $80 \mathrm{ml}$ of the fluorescence marker solution, and the mixture was gently stirred for $24 \mathrm{hr}$. The suspension was centrifuged at 2,588 $\times \mathrm{g}$ for $20 \mathrm{~min}$, and the supernatant was decanted to remove free FITC-dextran. To get rid of residual free FITC - dextran, the microgels were resuspended in HEPES ( $3 \mathrm{mM}, \mathrm{pH} 6$ ) and they were retrieved by centrifugation. The sediment of microgels was lyophilized for the release experiment. The amount of FITC-dextran loaded in microgels was determined by subtracting the amount of dye in the supernatant from the amount ( $35 \mathrm{mg}$ ) of dye added to HEPES buffer used for the hydration of dry microgel. The fluorescence intensity was measured at $520 \mathrm{~nm}$ with the excitation wavelength of $495 \mathrm{~nm}$. The amount of dye in the supernatant was determined using a calibration curve set up at $\mathrm{pH}$ 6.0. The amount of FITC-dextran loaded in microgel was expressed as specific loading, which is defined as mass of loaded dye per unit mass of dry microgel.

\section{7. pH-dependent Release from Microgels}

Release of FITC-dextran from microgels was investigated with varying pHs. Dry microgels, $0.2 \mathrm{~g}$, were put in $35 \mathrm{ml}$ of buffer solution contained in a $50 \mathrm{ml}$ beaker, and the suspension was gently stirred for $4 \mathrm{hr}$. MES was used for $\mathrm{pH} 3.5, \mathrm{pH} 4.0, \mathrm{pH} 5.0$, HEPES for $\mathrm{pH}$ 6.0, $\mathrm{pH} 7.0, \mathrm{pH} 8.0$, and Tris buffer for $\mathrm{pH}$ 9.0. An aliquot amount of suspension was taken at a given time and the suspension was filtered using a syringe filter. The fluorescence dye released was quantified by measuring the fluorescence intensity of the filtrate. The $\%$ release is defined as the amount released at a given time versus the amount loaded in the microgels at time 0 .

\section{RESULTS AND DISCUSSION}

\section{FTIR of Microspheres and Microgels}

Fig. 1 shows the FTIR spectra of uncrosslinked microspheres, soft microgels and hard microgels. In the spectrum of uncrosslinked microsphere (a), the peak observed at $1,557 \mathrm{~cm}^{-1}$ is due to the amino groups of chitosan, and the peak at $1,644 \mathrm{~cm}^{-1}$ is attributed to the carbonyl group of the acetyl amino group. In the spectrum of hard microgel (c), the peak observed at $1,552 \mathrm{~cm}^{-1}$ is due to the amino groups of chitosan, and the signal was smaller than that of the amino groups of uncrosslinked microsphere. This is possibly because some of amino groups participated in the condensation reaction with GA. The peak observed at $1,629 \mathrm{~cm}^{-1}$ is ascribed to $\mathrm{N}=\mathrm{C}$ - produced by the condensation reaction between the amino group of chitosan and

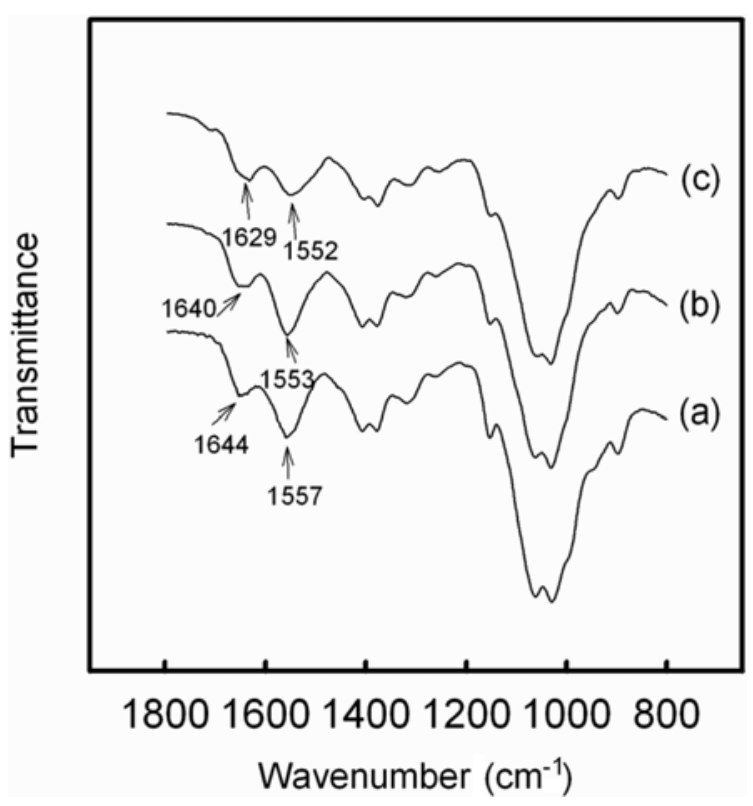

Fig. 1. FTIR spectra of uncross-kinked microspheres (a), soft microgels (b) and hard microgels (c). 
the aldehydric group of GA [26]. There was no outstanding difference between the spectrum of uncrosslinked microspheres (a) and that of soft microgel (b). The small amount of crosslinker (GA) used for the preparation would make difficult the detection of the crosslinking reaction.

\section{SEM of Microspheres and Microgels}

Fig. 2 shows SEM photos of uncrosslinked microsphere (a), soft microgels (b), and hard microgels (c). The microspheres and the microgels were few microns in diameter. The typical size of microparticles obtained by a spray-drying method was reported to be 1$10 \mu \mathrm{m}$ [27]. The surface of uncrosslinked microspheres was uneven and the microspheres looked like a golf ball. The surfaces of micro-
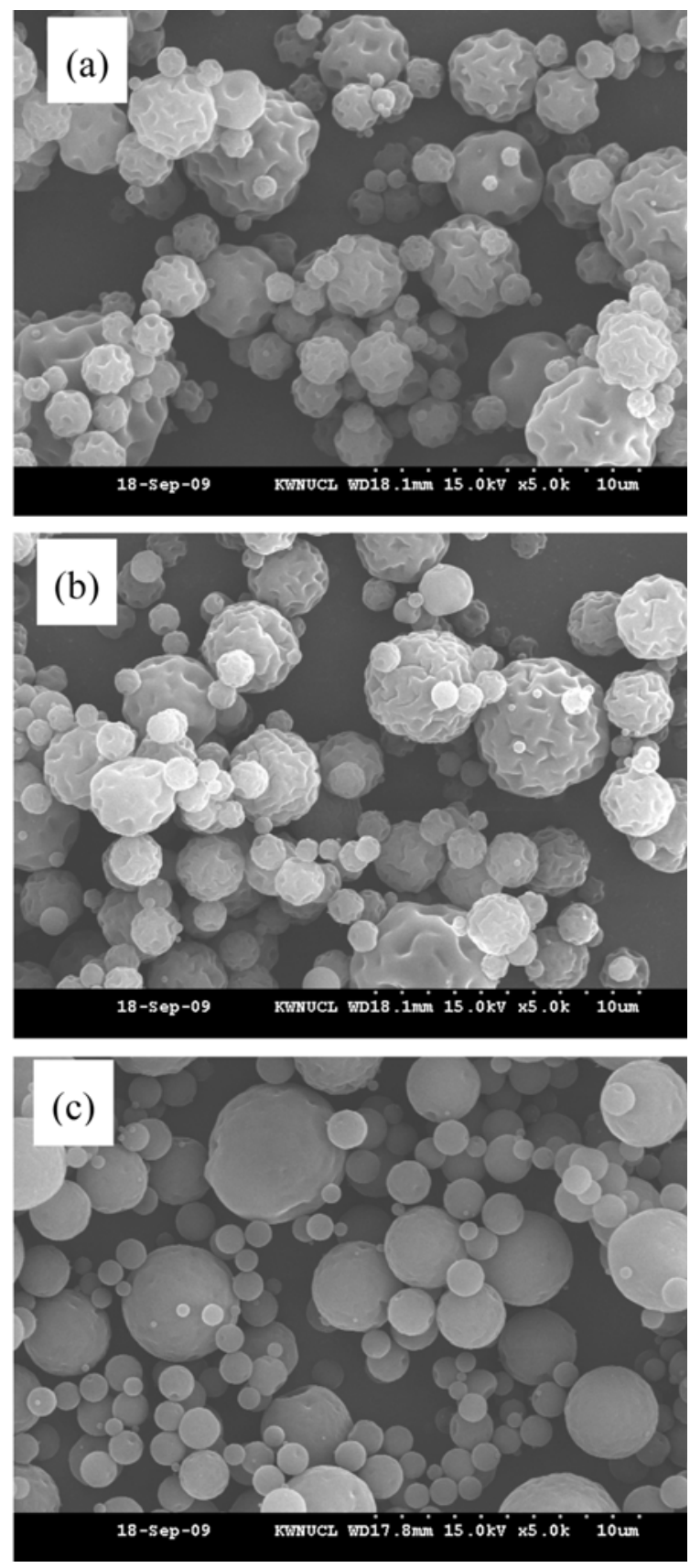

Fig. 2. SEM photos of uncross-linked microspheres (a), soft microgels (b), and hard microgels (c).

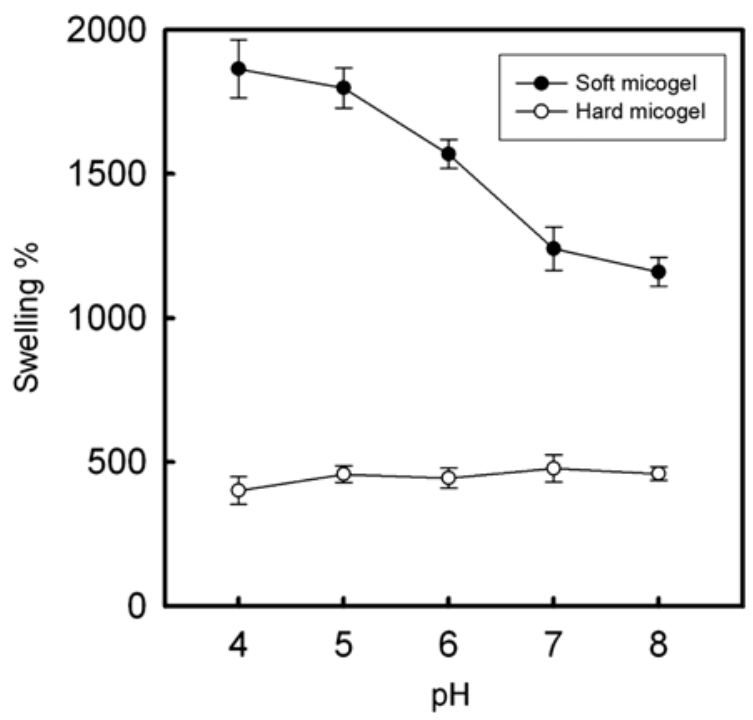

Fig. 3. Swelling ratios of soft microgels $(O)$ and hard microgels (O). All measurements were conducted in triplicate and averaged.

gels, especially hard microgel, were much more even than those of uncrosslinked microspheres. The cross-linking of chitosan will increase the mechanical stability of the microparticles. Accordingly, uncrosslinked microspheres would be subject to a surface deformation during a spray drying process.

\section{Swelling Ratio of Microgels}

Fig. 3 shows the swelling ratios of soft microgel and hard microgel. The swelling ratio of soft microgel decreased from $1,765 \%$ to $1,230 \%$ when the $\mathrm{pH}$ increased from 4.0 to 9.0. Under an acidic condition, the protonation of amino groups of chitosan will develop an electrostatic repulsion within the matrix of microgel. Due to the electrostatic force, the polymer chains constituting microgel will push each other away so the microgel would swell. Under an alkali condition, the deprotonation will mitigate the repulsion so the microgel would de-swell. Hard microgel, however, is $\mathrm{pH}$-insensitive in terms of swelling ratio. For example, swelling ratios at $\mathrm{pH} 4.0, \mathrm{pH}$ 5.0, $\mathrm{pH} 6.0, \mathrm{pH} 7.0, \mathrm{pH} 8.0$, and $\mathrm{pH} 9.0$ were $372 \%, 407 \%, 430 \%$, $450 \%$ and $440 \%$, respectively. The matrix of hard microgels would be rigid due to a high crosslinking density. Thus, the swelling ratio could be almost invariable whether the amino groups of chitosan were protonated or deprotonated. In addition, the swelling ratios of hard microgels at all $\mathrm{pH}$ tested were much smaller than those of soft microgels. This is also ascribed to a high crosslinking density of the hard microgels.

\section{Loading of FITC-dextran in Microgels}

The specific loading of FITC-dextran in soft microgel and hard microgel was $0.014 \mathrm{~g} / \mathrm{g}$ and $0.011 \mathrm{~g} / \mathrm{g}$, respectively. The higher specific loading of dye in soft microgel is possibly due to the higher swelling ratio of soft microgel. The swelling ratio is a measure of the capability of microgel to imbibe aqueous phase so a higher swelling will give a rise to a higher specific loading of a water soluble compound.

\section{5. pH-dependent Release from Microgels}

Fig. 4(a) shows the $\mathrm{pH}$-dependent release profiles of FITC dextran from soft microgels. In the full range of $\mathrm{pH}$ tested, the releases 

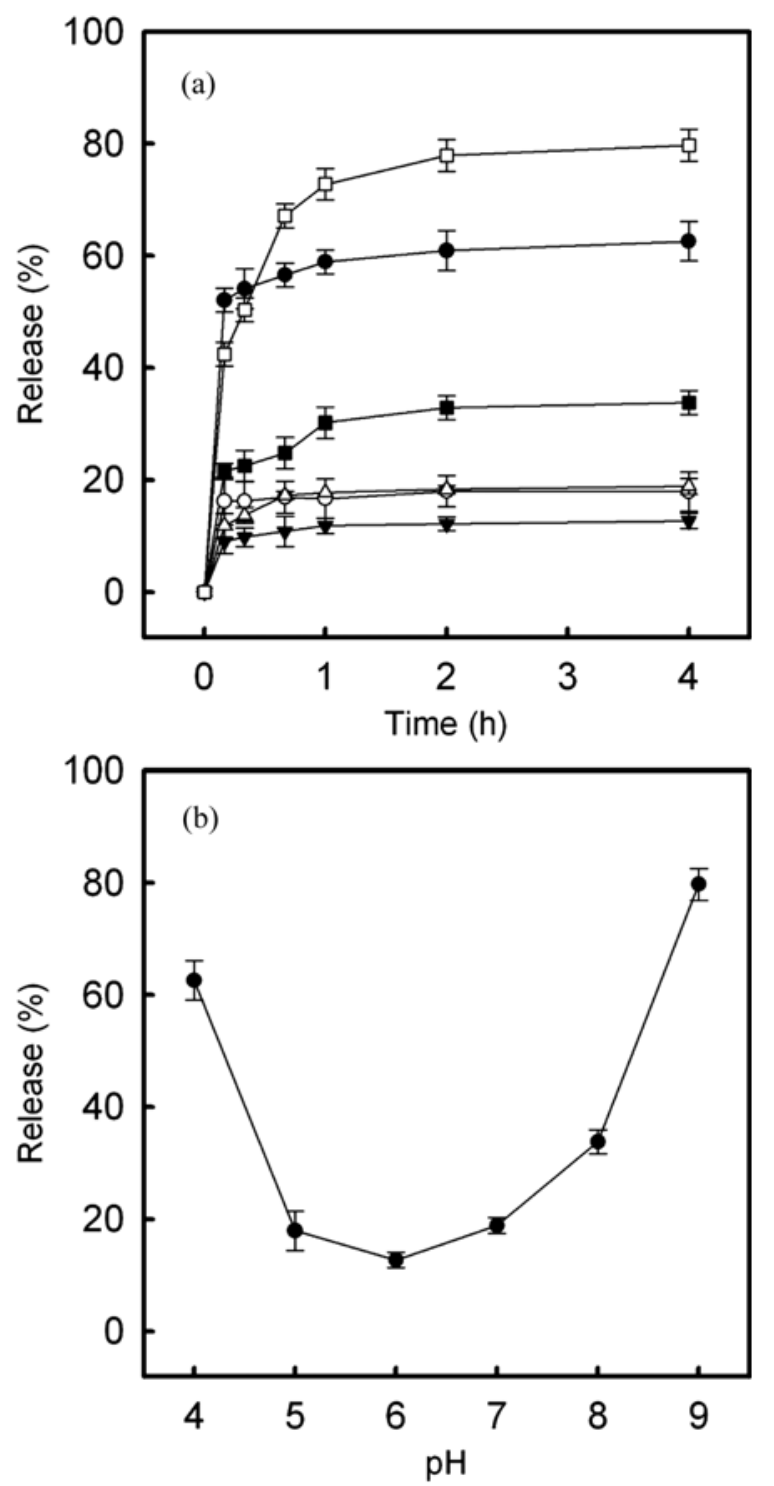

Fig. 4. Release profiles of FITC-dextran from soft microgels at $\mathbf{p H}$ $4.0(\bigcirc)$, pH $5.0(\bigcirc)$, pH $6.0(\nabla)$, pH 7.0 $(\triangle)$, pH $8.0(\square)$, and pH 9.0 ( $\square$ ) (a). Degree of release in $4 \mathrm{hr}$ at each pH (b). All measurements were conducted in triplicate and averaged.

increased in a saturation manner and they were almost completed in $2 \mathrm{hrs}$. That is, the releases seemed to follow a first order [28]. In case the drug carrier is a matrix type like microgels, the drug in the out-layer will be first depleted, and then that in the inner part will be. Accordingly, the area for mass transfer will decrease with time. Moreover, in order to release out from the inner part of microgels in the later stage of release, a drug is required to diffuse through the outer part of the microgel. Accordingly, as time is going on, the drug will travel through a longer path and it will encounter a greater diffusion resistance. In addition, the rate of mass transfer will be slackened down with time since the release medium was a finite system and it was not in a sink condition. The above-mentioned sentences may account for the first-order release. Fig. 4(b) shows the degrees of release in $4 \mathrm{hr}$. The degree of release decreased from
$65 \%$ to $12 \%$, when the $\mathrm{pH}$ of medium increased from 4.0 to 6.0 . In a strong acidic condition, most of amino groups of chitosan will be protonated, and the microgel tends to swell due to an electrostatic repulsion. In fact, the swelling ratio was higher at a lower $\mathrm{pH}$ (Fig. 3). Since the mesh size of the microgel is proportional to the swelling ratio, FITC-dextran would readily diffuse out of the microgel in a strong acidic condition (e.g., $\mathrm{pH} 4.0$ ). On the contrary, the degree of release increased from $12 \%$ to $82 \%$ when the $\mathrm{pH}$ of medium increased from 6.0 to 9.0. Despite the fact that the swelling ratio still decreased with increasing $\mathrm{pH}$ in the range of $\mathrm{pH}$ 6.0-9.0, how could the degree of release increase with increasing $\mathrm{pH}$ ? The fluorescence dye is believed to be released mainly by a squeezingout in the range of $\mathrm{pH}$ 6.0-9.0. In the lower $\mathrm{pH}$ range (4.0-6.0), diffusion through the meshes of the microgel seemed to control the degree of release. This could account for why the degree of release
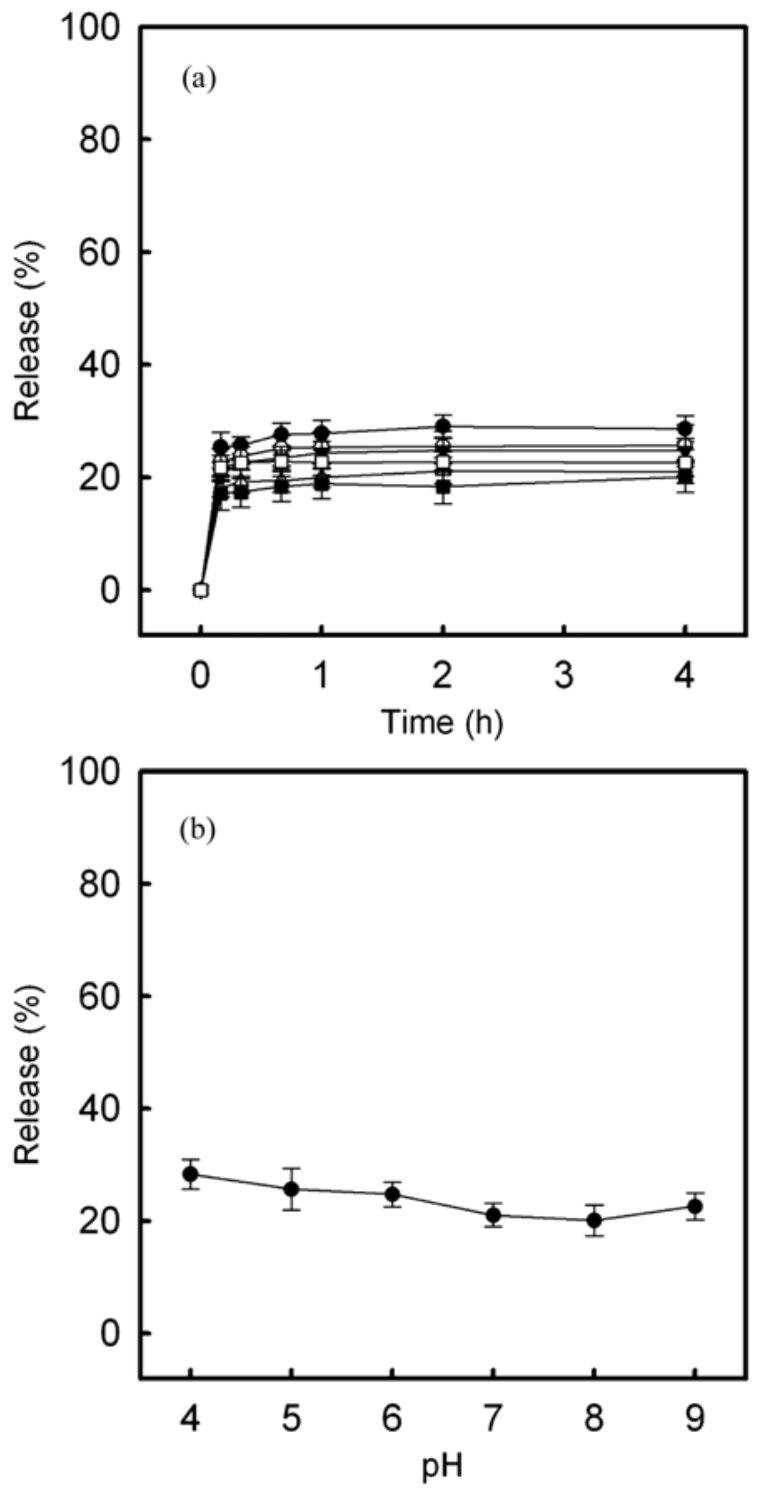

Fig. 5. Release profiles of FITC-dextran from hard microgels at pH $4.0(\bigcirc)$, pH $5.0(\bigcirc)$, pH $6.0(\nabla)$, pH $7.0(\triangle)$, pH 8.0 ( $\square)$, and pH 9.0 ( $\square$ ) (a). Degree of release in $4 \mathrm{hr}$ at each pH (b). All measurements were conducted in triplicate and averaged. 
decreased with increasing $\mathrm{pH}$. As the $\mathrm{pH}$ of the release medium increased further from $\mathrm{pH} 6.0$ to $\mathrm{pH} 9.0$, the swelling ratio also decreased further. In this circumstance, the content of the microgel could be squeezed out due to the de-swelling of the microgel. The microgel was initially in a dry state. However, wetting, de-swelling and squeezing out could occur in series. Fig. 5(a) shows the pHdependent release profiles of FITC-dextran from hard microgels. The release profiles are similar to those of soft microgels, but the degrees of releases at all $\mathrm{pHs}$ tested are much less than those from soft microgels. The crosslinking density of hard microgels would be much higher than that of soft microgels (chitosan to GA ratio of hard microgels was $1: 0.67$ and that of soft microgels was $1: 0.08$ ). As a result, the swelling ratio of hard microgels was much lower than that of soft microgels at all pHs tested (Fig. 3). The mesh size of hard microgels would be smaller due to the lower swelling ratio, and the smaller mesh could hinder the mass transfer through the matrix, leading to a suppressed release from hard microgels. In addition to the lower swelling ratio of hard microgel, the lower specific loading may also contribute to the lower releases, because thermodynamic activity of FITC-dextran in microgel will be proportional to the specific loading. Fig. 5(b) shows the degrees of release in $4 \mathrm{hr}$. The degree of release gradually decreased from $29 \%$ to $20 \%$ when the $\mathrm{pH}$ of release medium increased from 4.0 to 9.0. At all $\mathrm{pHs}$ tested, the swelling ratios were relatively small, compared with those of soft microgels, and they were not as $\mathrm{pH}$-sensitive as the swelling ratios of soft microgels were (Fig. 3). In this circumstance, the squeezing-out would hardly occur. Instead, diffusion through the matrix of hard microgels is believed to control the degree of release. Since the mesh size will decrease with increasing $\mathrm{pH}$, the rate of the diffusion will be slackened down with increasing $\mathrm{pH}$. This may be a reason why the degree of release gradually decreased with increasing $\mathrm{pH}$. Not only the cross-linking density but also the number of amino group of microgel would contribute to the $\mathrm{pH}$-sensitivity. The number of amino groups in hard microgel will be less than that of amino group in soft microgel, so it may also be responsible for the less $\mathrm{pH}$-sensitivity of hard microgel.

FITC-dextran $(40,000)$ was used as a model for a biologically active compound of high molecular weight. Wounded tissue and exudates are known to be acidic [29], so the chitosan microgels could be used as vehicles to deliver an electrically neutral active compound for wound healing.

\section{CONCLUSION}

Chitosan microgels were successfully prepared by a spray-drying method using GA as a cross-linker. Soft microgel exhibited a $\mathrm{pH}$-sensitive swelling ratio and a $\mathrm{pH}$-sensitive release of FITC dextran. The degree of release from the soft microgels decreased with increasing $\mathrm{pH}$ in the range of $\mathrm{pH} 4.0-6.0$, but it increased with increasing $\mathrm{pH}$ in the range of $\mathrm{pH}$ 6.0-9.0. In the acidic region, the release seemed to be controlled by diffusion, but, in the neutral and alkali region, the release was likely controlled by a squeezing-out mechanism. On the other hand, hard microgels showed a $\mathrm{pH}$-insensitive swelling ratio and a $\mathrm{pH}$-insensitive release, possibly due to the rigid matrix. The $\mathrm{pH}$-dependent degree of release could be controlled by varying chitosan to GA ratio (cross-linking density).

\section{ACKNOWLEDGEMENT}

This work was carried out with the support of "Cooperative Research Program for Agriculture Science \& Technology Development (Project No. PJ0074792011)" Rural Development Administration, Republic of Korea.

\section{REFERENCES}

1. D.-Y. Xu, G-J. Li, Z.-F. Liao and X.-H. He, Polym. Bull., 62, 183 (2009).

2. M. V. Risbud, A. A. Hardikar, S. V. Bhat and R. R. Bhonde, J. Control Release, 68, 23 (2000).

3. K. S. Soppimath, A. R. Kulkarni and T. M. Aminabhavi, J. Control Release, 75, 331 (2001).

4. M. K. Kang and J.-C. Kim, Colloid Polym. Sci., 288, 265 (2010).

5. Y. H. Kim, Y. H. Bae and S. W. Kim, J. Control Release, 28, 143 (1994).

6. H. Feil, Y. H. Bae and S. W. Kim, Macromolecules, 25, 5528 (1992).

7. A. Gutowska, Y. H. Bae, J. Feijen and S. W. Kim, J. Control Release, 22, 95 (1992).

8. C. Alvarez-Lorenzo, L. Bromberg and A. Concheiro, Photochem. Photobiol., 85, 848 (2009).

9. N. Yui, T. Okano and Y. Skurai, J. Control Release, 26, 141 (1993).

10. Y. Qiu and K. Park, Adv. Drug Deliv. Rev., 53, 321 (2001).

11. S. J. Murdan, J. Control Release, 92, 1 (2003).

12. H. Li, Z. Yuan, K. Y. Lam, H. P. Lee, J. Chen, J. Hanes and J. Fu, Biosens. Bioelectron., 19, 1097 (2004).

13. J. Connor and L. J. Huang, Cell Biol., 101, 582 (1985).

14. D. C. Drummond, M. Zignani and J.-C. Leroux, Prog. Lipid Res., 39, 409 (2000).

15. C.-J. Chu, J. Dijkstra, M.-Z. Lai, K. Hong and F. C. Szoka, Pharm. Res., 7, 824 (1990).

16. M. L. Huguet, R. J. Neufeld and E. Dellacherie, Process Biochem., 31, 347 (1996).

17. C. M. Silva, A. J. Ribeiro, M. Figueiredo, D. Ferreira and F. Veiga, AAPS J., 7, E903 (2005).

18. S. Simões, J. N. Moreira, C. Fonseca, N. Düzgüne and M. C. P. Lima, Adv. Drug Deliv. Rev., 56, 947 (2004).

19. S.-M. Jo, H. Y. Lee and J.-C. Kim, Int. J. Biol. Macromol., 45, 421 (2009).

20. R. Pelton, Adv. Colloid Interface Sci., 85, 1 (2000).

21. J. K. Oh, D. I. Lee and J. M. Park, Prog. Polym. Sci., 34, 1261 (2009).

22. H. Zhang, S. Mardyani, W. C. W. Chan and E. Kumacheva, Biomacromolecules, 7, 1568 (2006).

23. S. Mansouri, P. Lavigne, K. Corsi, M. Benderdour, E. Beaumont and J. C. Fernandes, Eur. J. Pharm. Biopharm., 57, 1 (2004).

24. M. Thanou, J. C. Verhoef and H. E. Junginger, Adv. Drug Deliv. Rev, 52, 117 (2001).

25. L. Zhang, Y. Jin, H. Liu and Y. J. Du, Appl. Polym. Sci., 82, 584 (2001).

26. Z.-X. Xue, G P. Yang, Z.-P. Zhang and B.-L. He, React. Funct. Polym., 66, 893 (2006).

27. J. K. Oh, D. I. Lee and J. M. Park, Prog. Polym. Sci., 34, 1261 (2009).

28. B. S. Rao and K. V. R. Murthy, Int. J. Pharm., 231, 97 (2002).

29. D. R. Williams, Coord. Chem. Rev., 151, 161 (1996). 